\title{
Solar disinfection of drinking water protects against cholera in children under 6 years of age
}

\author{
R M Conroy, M E Meegan, T Joyce, K McGuigan, J Barnes
}

\begin{abstract}
Background and Aims-We have previously reported a reduction in risk of diarrhoeal disease in children who used solar disinfected drinking water. A cholera epidemic, occurring in an area of Kenya in which a controlled trial of solar disinfection and diarrhoeal disease in children aged under 6 had recently finished, offered an opportunity to examine the protection offered by solar disinfection against cholera.

Methods-In the original trial, all children aged under 6 in a Maasai community were randomised by household: in the solar disinfection arm, children drank water disinfected by leaving it on the roof in a clear plastic bottle, while controls drank water kept indoors. We revisited all households which had participated in the original trial.
\end{abstract}

Results-There were 131 households in the trial area, of which 67 had been randomised to solar disinfection (a further 19 households had migrated as a result of severe drought). There was no significant difference in the risk of cholera in adults or in older children in households randomised to solar disinfection; however, there were only three cases of cholera in the 155 children aged under 6 years drinking solar disinfected water compared with 20 of 144 controls.

Conclusions-Results confirm the usefulness of solar disinfection in reducing risk of water borne disease in children. Point of consumption solar disinfection can be done with minimal resources, which are readily available, and may be an important first line response to cholera outbreaks. Its potential in chorine resistant cholera merits further investigation. (Arch Dis Child 2001;85:293-295)

Keywords: solar disinfection; cholera

In 1996, we published the results of a 12 week randomised field trial of solar disinfection of drinking water in 206 Maasai children aged 5 to 15. Children whose mothers kept their drinking water on the roof of the family home in transparent plastic bottles in the sunlight had a $10 \%$ reduction in the incidence of all diarrhoea, including a $24 \%$ reduction in the incidence of severe diarrhoea compared with control children whose mothers kept their drinking water indoors. ${ }^{1}$

We subsequently set up a larger study to extend the findings to children aged under 6 .
This study ended in September 1997. In October of 1997 a three year drought in the region ended with prolonged rains which brought flooding and disease outbreaks. Between about November 1997 and January 1998 there was an outbreak of cholera affecting adults and children in several areas of Kajiado District, including the areas of Embolei, Mutarurar, Eremit, and Singiraine, in which the solar disinfection trial had been run. Since all the families were continuing to use solar disinfection as they had during the trial, in addition to providing information on solar disinfection and risk of diarrhoeal disease, the cholera outbreak offered a unique opportunity to examine the protective effect of solar disinfection on the risk of cholera. Though no bacteriological investigation of the cholera outbreak was carried out at the time, it has remained endemic in the area, and subsequent investigation in early 1999 by Ministry of Health officials confirmed the presence of cholera bacteriologically.

\section{Methods}

We have described the methods in detail previously. ${ }^{12}$ All families with children under 6 years of age living in an area of approximately 400 square kilometres of Kajiado District extending southwards from Taporu towards Lake Magadi were randomised to either solar disinfection or control conditions. All households were issued with sufficient 1.5 litre clear plastic bottles to ensure an adequate supply of drinking water for all children under 5 . In the solar disinfection condition, mothers were instructed to keep the supply of drinking water for her children aged under 5 in the plastic bottles on the roof; in control households the mother was instructed to keep the water indoors in plastic bottles. All children aged under 5 were instructed to drink exclusively from these water supplies. Randomisation was by alternate household.

All households who had participated in the study were visited within six weeks of the outbreak by Maasai health workers or one of us (MEM) who speaks fluent Maasai and has worked in the area for the past 20 years. Because there are virtually no medical facilities accessible to the households in the study group, it was unlikely that any of the cases would have been investigated bacteriologically. It was therefore necessary to establish a verbal diagnosis based on translation into Maasai of the clinical features of cholera. ${ }^{3}$

Mothers and, where necessary, grandmothers were interviewed to establish if anyone in the household had been seriously ill during the period of the outbreak. All interviews were 
completed within eight weeks of the end of the outbreak. In the case of any person who had been seriously ill with diarrhoeal disease of sudden onset, they, or their mother (in the case of children) were asked what the illness had been. Detailed questions were asked in relation to all cases of voluminous, watery diarrhoea. Cholera was diagnosed when the case met these criteria:

(1) It was described using the specific Maasai word for cholera used by the clan of the person being interviewed.

(2) It included the three typical features of cholera recognised by the Maasai:

enkalokani-a voluminous, dehydrating diarrhoea which is very watery, has a characteristic "cholera" smell, a slightly bitter taste (aishamisham), and the texture of recently soured milk, or of a watery paste made from ochre

nepuk - a marked reduction in the amount of urine, often accompanied by changes in complexion, with the eyes becoming sunken and the skin wrinkled

nerrutoisinyi - literally "the calf muscles turn inside out"; cramps in the the calf muscles associated with diarrhoea.

The illness had to meet all four criteria to be rated as cholera. The Maasai terms used to describe the illness were recorded. Cholera in children aged under 3 was only diagnosed if the child presented with a typical history and there had been at least one definite case in the child's household.

Analysis was carried out on an intention to treat basis, with households classified as using solar disinfection or control on the basis of their original randomisation. Although all householders claimed to have continued to treat the drinking water for young children as instructed, no objective assessment of compliance was possible, as no field worker visited the families during the period of the outbreak. For this reason, the protective effect presented here must be regarded as a minimal estimate. However, as the protective effect was observed in free-living subjects, it may be regarded as evidence of the public health utility of solar disinfection in cholera risk reduction.

Because the use of solar disinfection had been randomised by household, robust variance estimators, implemented in Stata's svy procedures were used to adjust variances and confidence intervals. Each household was entered as a cluster (primary sampling unit). In addition, because there was considerable variation in risk of cholera between the seven locations involved in the study, the analysis was stratified by location. Logistic regression, with variance adjustment for the clustered design (children randomised by household), implemented in Stata's procedure svylogit, was used to express the relation of risk of cholera to use of solar disinfection in children under $6 .{ }^{4}$

\section{Results}

We recorded data on 131 households containing 299 children who had participated in the diarrhoeal disease study and were therefore aged under 6 years in September 1997. A further 19 households, containing 50 children who had participated in the diarrhoeal disease trial, had moved out of the area as a result of the drought which had affected the region for the previous three years.

Cholera meeting the definition above occurred in 31 households. In 24 households, both adults (persons aged over 15) and older children (aged 6-15) had been affected; in six households older children only were affected and in one household adults only were affected. Two elderly adults and two children aged 6-15 died.

Sixty seven of the 131 households were using solar disinfection at the time of the cholera outbreak. Cholera occurred in children aged 6-15 in $16(23.9 \%)$ of these households, compared with $14(21.9 \%)$ control households (relative risk $1.09,95 \%$ CI 0.58 to 2.05 ). Cholera in adults occurred in $14(20.9 \%)$ of the households using solar disinfection and in 11 $(17.2 \%)$ control households (relative risk 1.2, $95 \%$ CI 0.59 to 2.5 ).

There were 155 children aged under 6 years in the households using solar disinfection and 144 children of that age in the control households. There were three cases of cholera in children using solar disinfected water and 20 in the control children, giving an odds ratio of $0.12(95 \%$ CI 0.02 to $0.65, \mathrm{p}=0.014)$.

\section{Discussion}

The occurrence of a cholera outbreak shortly after the end of a formal trial of solar disinfection offers a unique opportunity to study the effect of solar disinfection in a way that would not have been practical or ethical as a prospective controlled trial, as the investigators would have been duty bound to begin preventive measures as soon as the outbreak occurred. The fact that only the younger children in each household were drinking solar disinfected water also allows a more stringent test of the effect, as it should not be present in older children or adults. The data, therefore, come as close to a randomised controlled design as is possible in studying cholera under the conditions under which it normally occurs.

The study data were collected by verbal report rather than substantiated by bacteriological investigation, which is the only realistic method of gathering data on cholera in much of sub-Saharan Africa, where even if patients travel as far as the district hospital, laboratory facilities for verifying the diagnosis may not be available. This is the case in the locations involved in the present study, where no facilities for identifying Vibrio cholerae in stools are available. It is notable, however, that cases have continued, and in March 1999 Ministry of Health officials investigated 43 cases identified by Maasai health workers, using the same case definitions used here, of whom 42 were confirmed bacteriologically as cases of cholera.

In fact, the Maasai are a people whose medical terminology is rich and precise in its taxonomy of diarrhoeal disease. They are familiar with cholera, which occurs in outbreaks in the area, and have a precise 
vocabulary to describe its key features in their language.

Though the diagnosis and management of severe cholera (cholera gravis) are well known, the problem of public health management is compounded by the fact that few patients infected will develop the severe form of the illness, ${ }^{5}$ and many will be asymptomatic. Effective public health measures have therefore to be taken at community level, and must include an emphasis on point of use disinfection as an important element in control and prevention. ${ }^{2}$ Solar disinfection offers the possibility of using low cost or zero cost interventions which can be put in place swiftly by the community as a first line of defence, without the need to await outside help, as empty plastic bottles are becoming ubiquitous, even in the poorest of areas. Furthermore, significant inactivation of bacteria occurs even in highly turbid water and at relatively low optical irradiances. ${ }^{6}$ Solar disinfection may offer a way of decreasing risk of infection while more permanent and effective solutions to the problem of water quality are being found. Further work is needed, however, to elucidate the role of solar disinfection in the inactivation of $C$ cholerae. MacKenzie and colleagues report that at high altitude (2850 metres) there is a considerable bactericidal effect of sunlight, while at 500 metres the effect is considerably less. ${ }^{7}$

There are also recent concerns about the development of a "rugose" form of $V$ cholerae which may resist chlorine disinfection. ${ }^{8}$ The results presented here, while not providing definitive evidence, certainly suggest that solar disinfection should be evaluated in cholera risk reduction as a rapid response to cholera outbreaks, and in the event of chlorine resistant cholera becoming a public health problem.

Finally, our study highlights the potential for using detailed knowledge of ethnic medical concepts to carry out field work in disease investigation. Far from being medically ignorant, traditional cultures have elaborate taxonomies of disease, which can be used to build up reliable verbal diagnostic and screening procedures. Such traditional medical knowledge is an under used resource in health promotion and disease investigation. Furthermore, the close integration of disease investigation with traditional medical frames of references can pave the way for credibility of results and adoption of preventive measures among the community.

This research was supported by a grant by the Royal College of Surgeons in Ireland.

1 Conroy RM, Elmore-Meegan M, Joyce T, et al. Solar disinfection of drinking water and diarrhoea in Maasai children: fection of drinking water and diarrhoea in Maasai

2 Conroy RM, Elmore-Meegan M, Joyce T, et al. Solar disinConroy RM, Elmore-Meegan M, Joyce T, et al. Solar disin-
fection of drinking water reduces diarrhoeal disease-an fection of drinking water reduces dia
update. Arch Dis Child 1999;81:337-8.

3 Sanchez JL, Taylor DN. Cholera. Lancet 1997;349:1825-30.

4 StataCorp. Stata statistical software, release 6.0. Volume 4 (Reference Su-Z). College Station, TX: Stata Corporation, 1999:18-30.

5 Gangarosa EJ, Mosely WH. Epidemiology and surveillance of cholera. In: Barrua D, Burrows W, eds. Cholera. Philadelphia: Saunders, 1974:381-403.

6 McGuigan KG, Joyce TM, Conroy RM, et al. Solar disinfection of drinking water contained in transparent plastic bottles: characterizing the bacterial inactivation process. F Appl Microbiol 1998;84:1138-48.

7 MacKenzie TD, Ellison RT III, Mostow SR. Sunlight and cholera. Lancet 1992;340:367.

8 Morris JG, Sztein MB, Rice EW, et al. Vibrio cholerae 01 can assume a chorine-resistant rugose survival form that is can assume a chorine-resistant rugose survival form
virulent for humans. F Infect Dis 1996;174:1364-8. 